Document downloaded from:

http://hdl.handle.net/10251/165761

This paper must be cited as:

Vidal Rodriguez, B. (2020). Broadband photonic microwave noise sources. IEEE Photonics Technology Letters. 32(10):592-594. https://doi.org/10.1109/LPT.2020.2986739

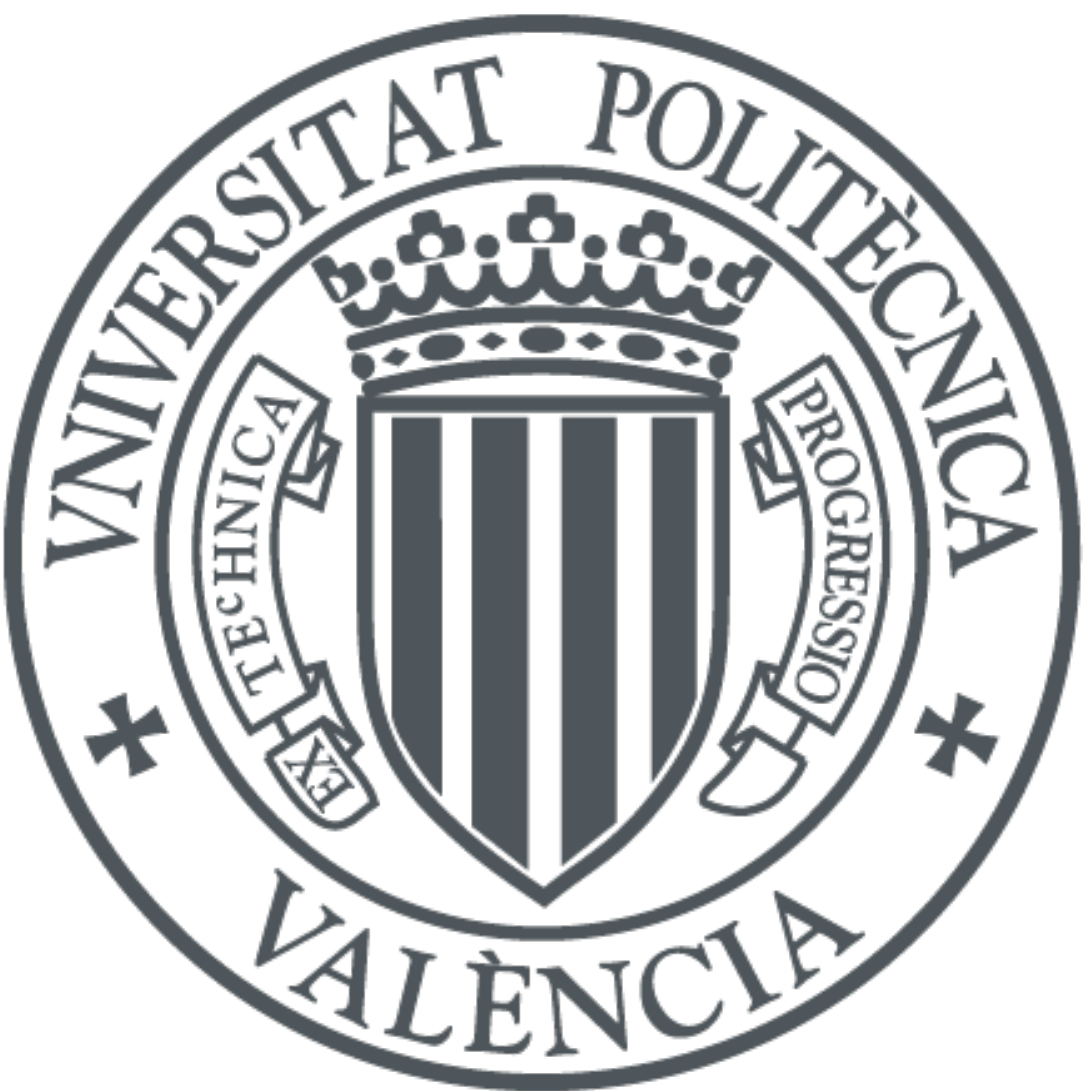

The final publication is available at

https://doi.org/10.1109/LPT.2020.2986739

Copyright Institute of Electrical and Electronics Engineers

Additional Information 


\title{
Broadband photonic microwave noise sources
}

\author{
Borja Vidal, Senior Member, IEEE
}

\begin{abstract}
The generation of wideband microwave white noise based on optical technology is investigated. It is shown that strong broadband noise in the microwave band can be generated using amplified spontaneous noise (ASE). Measurements show wideband operation $(0-20 \mathrm{GHz})$ and very large tunable excess noise ratio (ENR) up to $62 \mathrm{~dB}$. Photonics offer an alternative implementation of noise sources for system characterization over very broad bandwidths and potential to be employed in the millimeter and submillimeter-wave bands where conventional noise sources are complex or inexistent.
\end{abstract}

Index Terms-Microwave photonics, amplified spontaneous noise, microwave instruments.

\section{INTRODUCTION}

$\mathrm{M}$ ICROWAVE photonics is an interdisciplinary field focused on applying photonic technology to build microwave and mm-wave components and systems. The rationale is exploiting some particular features of fiber optics to enhance the performance of microwave systems. Photonics provide features such as large bandwidth, low and frequency independent propagation losses and potential for integration. From the original concept of remote signal delivery [1], other applications were developed such as optical beamforming [2], photonic microwave filters [3], and signal measurement [4], just to cite some of the most widely researched applications.

Here, a new application of microwave photonics is investigated, the use of photonic technology for the implementation of microwave noise sources. A microwave noise source [5-6] is a device that produces white noise in a controlled way. These sources are useful for the measurement of the frequency response of microwave components and, especially, for the measurement of their noise figure, which is frequently measured through the Y-factor method [7]. This method relies on measuring the ratio at the output of the device under test of two noise power levels, one measured with the noise source on and another with the noise source off.

Microwave noise generators are commonly based on electronic devices which rely on two fundamental processes: thermal noise and shot noise. Devices relying on the former are based on hot/cold loads, whereas those relying on shot noise include gas-discharge tubes, vacuum diodes, or solid-state noise diodes. Here, unlike previous approaches, a microwave noise source based on amplified spontaneous emission (ASE) noise [8] is explored. Photonic-based implementations can provide

Submitted $25^{\text {th }}$ February 2020 . This work was supported in part by the European Union's Horizon 2020 research and innovation programme (ULTRAWAVE-project, no. 762119). controlled levels of very wideband noise which will be just limited by the bandwidth of the photodiode. It means that this approach has potential for the implementation of noise generators for component characterization up to $100 \mathrm{GHz}$ and using unitravelling photodiodes (UTC-PD) up into the sub-THz region, where there is a lack of compact noise sources.

\section{THEORY}

Microwave noise sources used to perform measurements following the Y-factor technique need to provide two different known levels of noise power, which are usually known as cold $\left(T_{c}\right)$ and hot $\left(T_{h}\right)$. When the noise generator is on the cold level, the output will be the thermal noise associated to the physical temperature of the source. When activated, the generator will produce a greater noise power. Thus, microwave noise sources are characterized by their excess noise ratio (ENR) which is given by [7],

$$
E N R=10 \cdot \log _{10}\left(\frac{T_{h}-T_{c}}{T_{0}}\right)
$$

where $T_{0}$ is $290 \mathrm{~K}$.

A photonic microwave noise source can be implemented using an ASE-source in combination with a photodiode with a bandwidth covering the microwave bands of interest, as shown in Figure 1. To avoid the saturation of the photodiode, the ASE spectrum is filtered and amplified before photodetection. It also avoids the saturation of the EDFA. As in conventional microwave sources, the cold state will be given by thermal noise, i.e. no injection of optical noise, whereas the hot state will be defined by the injection of ASE noise to the photodiode and the domination at its output of the spontaneousspontaneous ASE noise contribution, which has been extensively studied, e.g. [8].

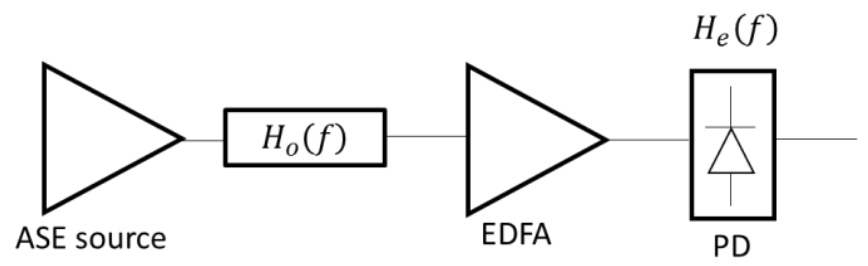

Fig. 1. Block diagram for the photonic microwave noise generator.

B. Vidal is with the Nanophotonics Technology Center, Universitat Politècnica de València, c/Camino de Vera, sn, 46022, Valencia, Spain. (e-mail: bvidal@dcom.upv.es). 
The output of the ASE source can be assumed to be white noise generated by the amplified spontaneous emission with a given power spectral density $\left(S_{0}\right)$. This noise is filtered by an optical filter given by the frequency response, $H_{0}(f)$, and then amplified by an Erbium-doped fiber amplifier (EDFA). Finally, it is detected in a square-law photodetector (PD), with a lowpass $\mathrm{RF}$ response that can be modelled with a frequency constant responsivity, $\mathcal{R}$, and the optoelectronic frequency response, $H_{e}(f)$.

The detected noises are thermal noise, ASE-ASE beat noise and ASE-shot noise. However, the ASE-ASE beat noise dominates over thermal and ASE-shot noises and they can be neglected [8]. The power spectral density (PSD) of the photocurrent noise generated from the photodetection of the ASE-ASE contribution is given by [9],

$S_{\text {out }}(f)=\mathcal{R}^{2} S_{0}^{2}(f)\left|H_{e}(f)\right|^{2} \int\left|H_{0}\left(f^{\prime}\right) \cdot H_{0}\left(f+f^{\prime}\right) \cdot\right|^{2} d f^{\prime}$

Assuming Gaussian shaped filters, it can be shown that the beat-noise limited signal to excess intensity noise ratio from a spectrum sliced incoherent optical source, i.e. the squared ratio between mean photocurrent and variance, is given by [10].

$$
S N R_{C W}=\frac{\langle i\rangle^{2}}{\sigma^{2}} \approx \frac{n_{p} B_{0}}{2 B_{e}}
$$

where $n_{p}$ is 1 for polarized light or 2 for unpolarized light and $B_{o}$ and $B_{e}$ are the equivalent noise bandwidth of the Gaussian filters, $H_{o}(f)$ and $H_{e}(f)$, respectively. Since the bandwidth of the photodiode is much smaller than the optical slicing filter, the photodiode will tend to saturate, generating a large DC current but not providing strong white electric noise at its output. To avoid PD saturation and maximize noise generation, the $S N R_{C W}$ has to be minimized which can be done by using an optical filter with a bandwidth slightly larger than the one of the photodiode, as expressed by (3).

\section{EXPERIMENTAL RESULTS}

A system following the block diagram shown in Fig. 1 has been implemented to experimentally demonstrate the feasibility of using incoherent optical sources to generate white electric noise. An ASE source with an output power of $12 \mathrm{dBm}$ is used to generate broadband optical noise. To have enough noise variance without a photocurrent with a large DC component, the broadband noise is spectrally sliced by a tunable Gaussian optical filter with a FWHM of $1 \mathrm{~nm}$. This filter was chosen by the availability in the laboratory of no other optical filter with a bandwidth closer to the photodiode bandwidth. Then, a preamplifier EDFA, Pritel FA-30, boosts the optical noise power before photodetection. The photodiode is a high power InGaAs PIN with a responsivity $(\mathcal{R})$ of 0.85 and a bandwidth of $20 \mathrm{GHz}$. Figure 2 shows the spectrum of the optical noise after being filtered by the optical filter $\left(H_{o}(f)\right)$ for different total optical noise power measured with a powermeter at the output of the preamplifier EDFA. A microwave spectrum analyzer has been used to measure the noise power at the output of the photodiode.

Figure 3 shows the measured electrical spectrum of the ASE-

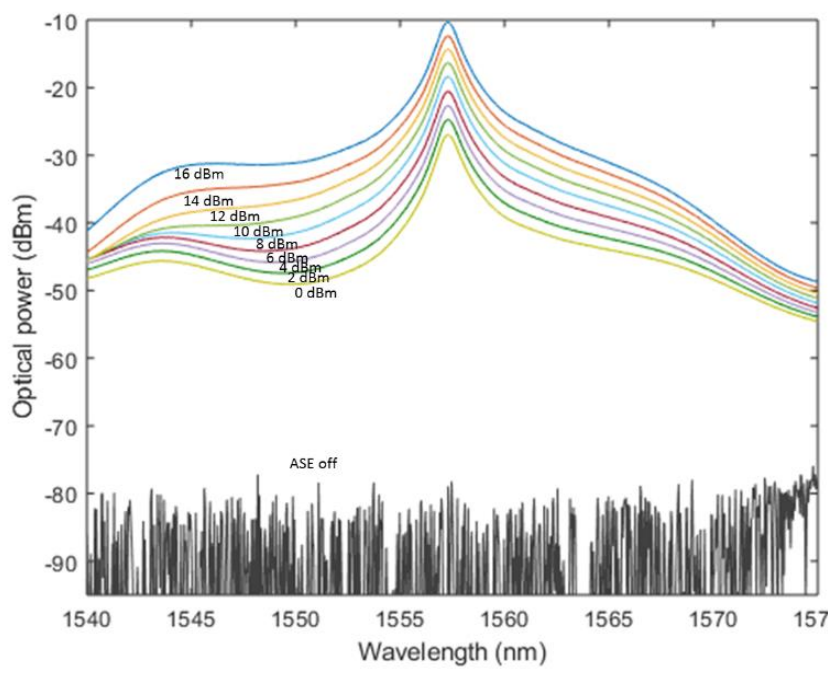

Fig. 2. Experimental optical spectrum of the amplified spontaneous emission after the optical filter for different total ASE powers.

ASE beat noise after photodetection for different values of total ASE power. Figure 4 shows the corresponding temporal signals. In both cases, the white noise is controlled through the gain of the EDFA preamplifier. It can be seen that broadband noise can be generated with the bandwidth limitation given by the photodiode $\left(H_{e}(f)\right)$. The flatness of the noise power is also given by the optoelectronic response of the photodiode since the optical filter is wider than the photodiode bandwidth.

If the ENR is higher than $15 \mathrm{~dB}$, it can be approximated as:

$$
E N R=-174 \frac{d B m}{H z}-S_{\text {meas }}[\mathrm{dBm} / \mathrm{Hz}]
$$

where $S_{\text {meas }}$ is the power spectral density of noise as measured with a microwave spectrum analyzer.

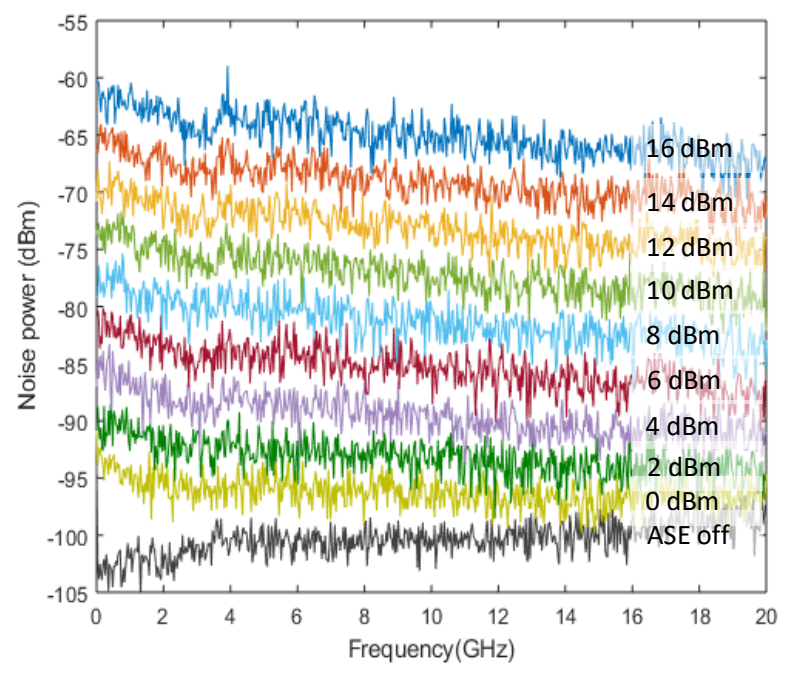

Fig. 3. Experimental measurement of noise power at photodiode output for different total ASE power levels. The resolution bandwidth is $100 \mathrm{kHz}$. 


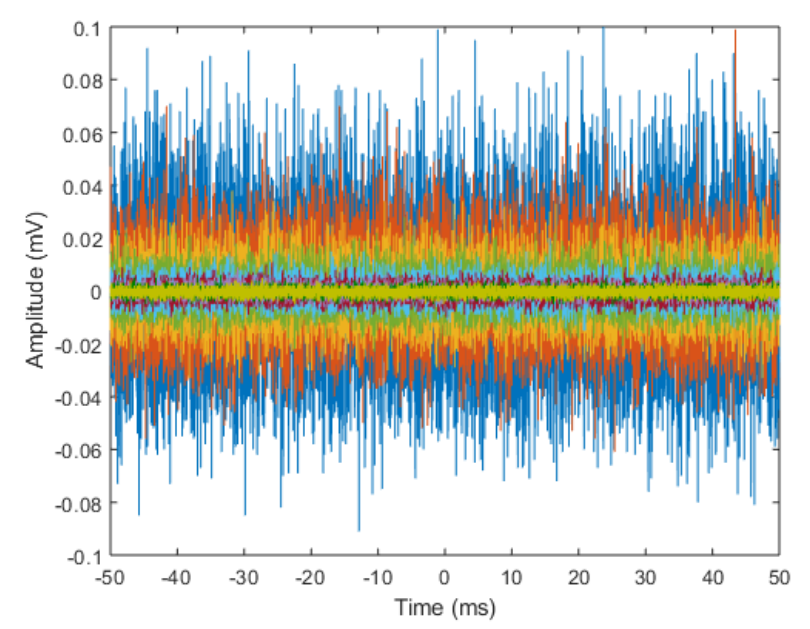

Fig. 4. Temporal traces of the noise waveforms for each ASE power level.

The ENR can be estimated from (4), which is shown in Fig. 5 . As it can be seen there is a good linear relation between total optical noise power and ENR $\left(R^{2}=0.9986\right)$.

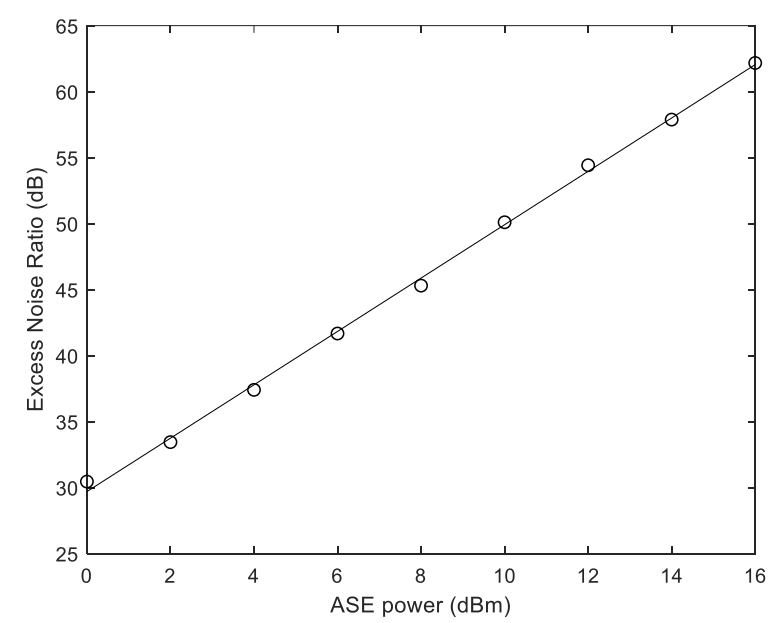

Fig. 5. Relation between total ASE noise power and ENR measured from PSD at $5 \mathrm{GHz}$.

Finally, the mismatch at the output of the PD for different power levels has been measured with a vector network analyzer. Figure 6 shows the $S_{11}$ parameter of the PD and its change with ASE power. The maximum change in $S_{11}$ is smaller than $2 \mathrm{~dB}$, i.e. a VSWR change of $2 \%$. Since the photonic scheme provides large ENR, a microwave attenuator can be introduced at the output of the photodiode to adapt ENR to optimum values. This, in turn, enhances the RF impedance matching at the output of the noise source. Tests with a $16 \mathrm{~dB}$ attenuator at the output of the PD shows changes in $\mathrm{S}_{11}$ smaller than $0.06 \mathrm{~dB}$ (VSWR changes smaller than 0.09\%). Given the strong excess noise that can be generated with this approach, very large attenuation is possible making impedance mismatches between hot and cold states even smaller.

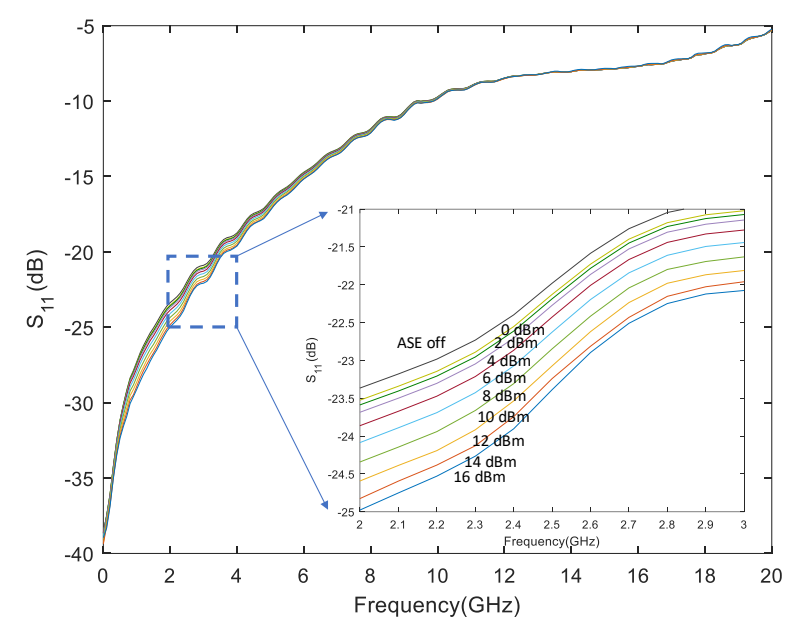

Fig. 6. Reflection coefficient $\left(S_{11}\right)$ at the output of the photodiode for different ASE total noise powers. (Inset) Detail between 1 and $3 \mathrm{GHz}$.

\section{CONCLUSION}

It has been experimentally shown that the thermal-like excess intensity noise derived from spectrum-sliced optical incoherent broadband sources can be used to implement microwave noise sources with large ENR. Thus, photonic technology can offer an alternative implementation of this microwave functionality. Microwave noise generators are basic components for the characterization of components and systems through the Yfactor method. The photonic implementation provides large bandwidth which will be given by the photodiode. Thus, this method enables the development of noise sources with potential to operate up to the sub- $\mathrm{THz}$ range, and flexibility to obtain large ENR, which are interesting for characterization of radiometers, radar systems and automated test equipment. This is a new application of photonic technology in the field of microwave photonics.

\section{REFERENCES}

[1] S.M. Foreman, K.W. Holman, D.D. Hudson, D.J. Jones, J. Ye, "Remote transfer of ultrastable frequency references via fiber networks", Review of Scientific Instruments, vol. 78, 021101, 2007.

[2] B. Vidal, T. Mengual, C. Ibáñez-López, J. Martí, "Optical Beamforming Network based on Fiber Optical Delay Lines and Spatial Light Modulators for Large Antenna Arrays", IEEE Photon. Technol. Lett., vol. 18, no. 24, pp. 2590-2592, 2006.

[3] B. Vidal, M. A. Piqueras, J. Martí, "Tunable and Reconfigurable Photonic Microwave Filter based on Stimulated Brillouin Scattering", Optics Letters, vol. 32, no. 1, pp. 23-25, 2007.

[4] B. Vidal, T. Mengual, J. Martí, "Photonic Technique for the Measurement of Frequency and Power of Multiple Microwave Signals", IEEE Trans. Microw. Theory Tech, vol., 58, no. 11, pp. 3103-3108, 2010.

[5] D. E. Susans, "Semiconductor-diode V.H.F. and U.H.F. noise sources", Electron. Lett., vol. 4, no. 4, pp. 72-73, 1968.

[6] S. Arslan, B. S. Yıldırım, "A Broadband Microwave Noise Generator Using Zener Diodes and a New Technique for Generating White Noise", IEEE Microw. Wirel. Compon. Lett., vol. 28, no. 4, pp. 329-331, 2018.

[7] Application note 57-2: "Noise Figure Measurement Accuracy: The Yfactor Method", Keysight Technologies, 2019.

[8] E. Desurvire, "Erbium-doped fiber amplifiers", Ed. Wiley, 1994.

[9] R.C. Steele, G.R. Walker, N.G. Walker, "Sensitivity of optically preamplified receivers with optical filtering", IEEE Photon. Technol. Lett., vol. 3, no. 6, pp. 545-547, 1991.

[10] J.S. Lee, "Signal-to-Noise ratio of spectrum-sliced incoherent light sources including optical modulation effects", J. Lightw. Technol., vol. 14, no. 10, pp. 2197-2201, 1996. 